\title{
Cyclic optical activation of semiconducting gas sensors: influence of cycling frequency
}

\author{
Danielle Klawinski ${ }^{1}$, Daniel Meixner ${ }^{2}$, Claus-Dieter Kohl ${ }^{2}$, Thorsten Wagner ${ }^{1}$ \\ ${ }^{1}$ Paderborn University, Department of Chemistry, Warburger Str. 100, 33098 Paderborn, Germany \\ 2 Justus-Liebig-University Giessen, Institute of Applied Physics, Heinrich-Buff-Ring 16, 35392 Giessen, \\ Germany \\ thorsten.wagner@upb.de
}

\begin{abstract}
Resistive semiconducting gas sensors are typically cost-efficient and highly sensitive devices. However, due to the detection mechanism based on oxidizing or reducing surface reactions they lack in inherent selectivity. To compensate this, different methods can be applied, as e.g. utilizing catalytic supplements or temperature cycled operation, each with its specific pros and cons. Here we present a new method based on the utilization of surface reaction kinetics by cyclic optical activation in the UV/Vis range. Optical activation enables low temperature operation of the sensing element and therefore reduces the power consumption of the element drastically. This work focuses on the systematic optimization process of the illumination cycle for detection of ozone on nanoporous indium oxide $\left(\mathrm{In}_{2} \mathrm{O}_{3}\right)$ sensing layer.
\end{abstract}

Key words: semiconducting gas sensors, cyclic optical activation, indium oxide, ozone sensing, reaction kinetics

\section{Introduction}

In the field of resistive semiconducting gas sensors increasing the selectivity or an enhancement of the sensor activity is of high interest. Resistive semiconducting gas sensors operate typically at high temperatures to speed up reaction kinetics because the sensor kinetics is generally slow without thermal activation. Alternatively, optical activation by light in the UV/Vis range affects the kinetics in sensing materials $[1,2]$.

As shown in literature, thermal activation affects the whole sensing layer which is in contrast to optical activation. In the case of optical activation only a surface-near region of the sensing layer is penetrated. This is why nanostructuring of the sensing layer and the sensing material influences optical properties and hence the sensors behaviour under illumination [3].

Indium oxide $\left(\ln _{2} \mathrm{O}_{3}\right)$ is a promising gas sensing material for optical activated low temperature $\left(<100^{\circ} \mathrm{C}\right)$ measurements of oxidizing gases like ozone.

It was already shown that continuous illumination of nanostructured $\operatorname{In}_{2} \mathrm{O}_{3}$ sensing layers significantly speeds up the sensor reaction as well as the regeneration times under exposure of ozone or $\mathrm{NO}_{2}[4,5]$. Cyclic illumination reduces the maximum response but improves the sensor kinetics further. It could be shown that especially the regeneration time of the cyclic illuminated sensor is only half of the value than for the continuously illuminated sensor under ozone exposure [6].

The cyclic illumination profile is causing an asymmetrical sawtooh shaped resistance change of the sensing layer which is due to electronic and photo-activation followed by relaxation processes of the material [6]. This non-equilibrium state of the sensing layer during cyclic operation can be utilized further to gain additional information on the time constants of the underlying chemical reactions. The resistance response of illumination cycles with varying durations and frequencies exhibits multi-signal properties comparable to temperature cyclic operation [7]. Simple mathematical data analysis allows e.g. signal stabilization; long term drift effects can be separated from gas induced signal changes.

In this work, we present a systematic optimization process of the illumination cycle to study the influence of cyclic illumination and chemical surface reaction during gas exposure on the sensing layer.

\section{Experimental}

Ordered mesoporous $\ln _{2} \mathrm{O}_{3}$ particles were synthesized by structure replication using mesoporous KIT-6 silica as structure matrix. The structure matrix was synthesized by sol-gel 
process. Details of the synthesis strategy and characterization can be found in literature [8].

The sensing layers were prepared by dropcoating of an aqueous dispersion of $\ln _{2} \mathrm{O}_{3}$ particles (25 mg particles in $1 \mathrm{~mL}$ dest. $\mathrm{H}_{2} \mathrm{O}$ ) onto commercially available sensor substrates (UST $\mathrm{GmbH}$, alumina based, $3 \times 3 \mathrm{~mm}$ with interdigitated electrodes and Pt10 heater). The sensing layers were dried at room temperature.

Resistive gas sensing measurements were carried out with ozone as test gas at a concentration of $500 \mathrm{ppb}$ at $150{ }^{\circ} \mathrm{C}$. For this a custom build gas mixing equipment based on mass flow controllers was utilized to supply the desired test gas concentration in synthetic air $(500 \mathrm{~mL} / \mathrm{min}$ flow rate) with $20 \%$ relative humidity. The sensing layer resistance was measured utilizing custom build electronics (potentiostatic mode at a voltage of $0.5 \mathrm{~V}$ ).

A blue LED (wavelength peak maximum at $466 \mathrm{~nm}$ ) was used for cyclic illumination of the sensor. The LED was mounted at a distance of $3 \mathrm{~mm}$ to the sensor (custom build PTFE housing) and driven by a precision current source (controlled by $\mu$-controller) [6]. Symmetric square shaped current pulses (amplitude of $80 \mathrm{~mA}$ ) were used for cyclic illumination. The cycling frequency was varied by using different illumination times (2, 5 and 10 seconds). For example, the sensor was illuminated for $2 \mathrm{~s}$ followed by a dark period of $2 \mathrm{~s}$.

\section{Results and discussion}

The synthesized $\ln _{2} \mathrm{O}_{3}$ particles exhibit pores with a diameter of 4,5 and $12 \mathrm{~nm}$ and a specific surface area of $100 \mathrm{~m}^{2} / \mathrm{g}$. The particle size is $170 \mathrm{~nm}$. Details of material characterization can be found in literature [8].

The sensor is illuminated by blue LED light using a square shaped current signal which leads to a sawtooth shaped resistance change of the sensing layer (schematic see Fig. 1). The resistance is decreasing during illumination, which is due to an electronic and photoactivation of the sensing layer. After turning off the illumination, the resistance is increasing because of relaxation processes.

The influence of the cyclic illumination on the sensor kinetics under ozone exposure was evaluated by comparing different illumination times (2, 5 and $10 \mathrm{~s})$. The results are shown in Fig. 2.

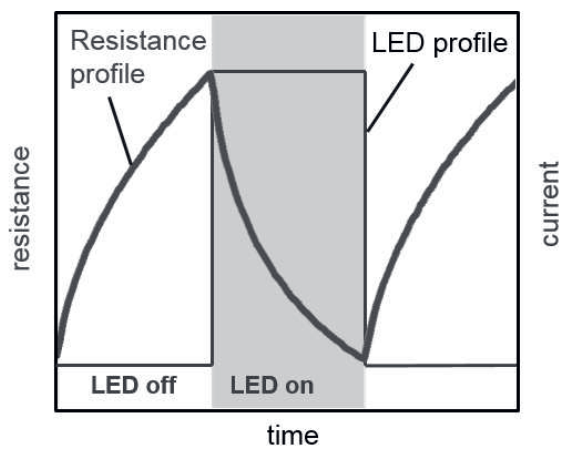

Fig. 1. Schematic of the square shaped LED profile and the resulting resistance profile of $\operatorname{In}_{2} \mathrm{O}_{3}$ particles.

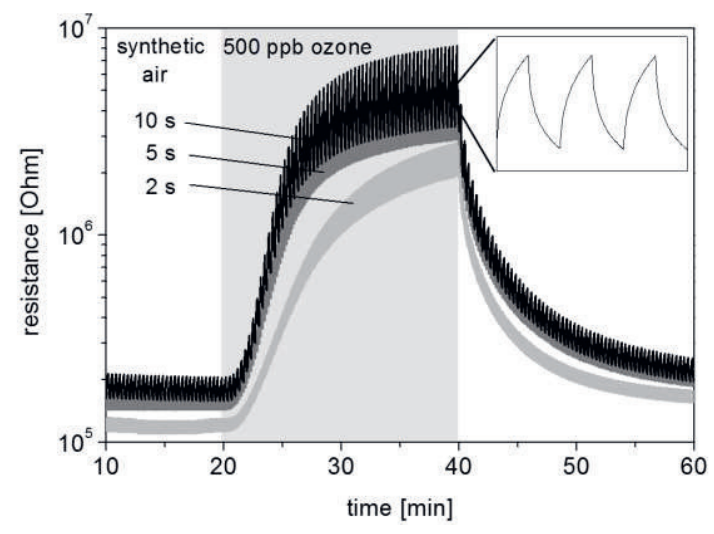

Fig. 2 Resistance vs. time of mesoporous $\ln _{2} \mathrm{O}_{3}$ under ozone exposure (500 ppb, $500 \mathrm{~mL} / \mathrm{min}$ flow rate, $20 \%$ relative humidity, $150^{\circ} \mathrm{C}$ ) for different illumination times with blue light (466 $\mathrm{nm})$.

The mean value of the resistance of the sensing layer is increasing with increasing illumination time (Fig. 2).

The cyclic operation mode enables a multisignal evaluation which can be used for further investigations.

The multi-signal evaluation, defining the sensor response as

$$
\text { response }=m_{1}-m_{0}
$$

enables the determination of the optimum illumination time (Fig. 3). $m_{0}$ and $m_{1}$ are measurement points in the illuminated part of the measured resistance signal (Fig. 3, inset). As can be seen (Fig. 3) the response of the sensor depends on the illumination time.

During ozone exposure, ozone molecules can adsorb and react on the $\ln _{2} \mathrm{O}_{3}$ surface. We assume that the different response signals are caused by these underlying surface reactions. 


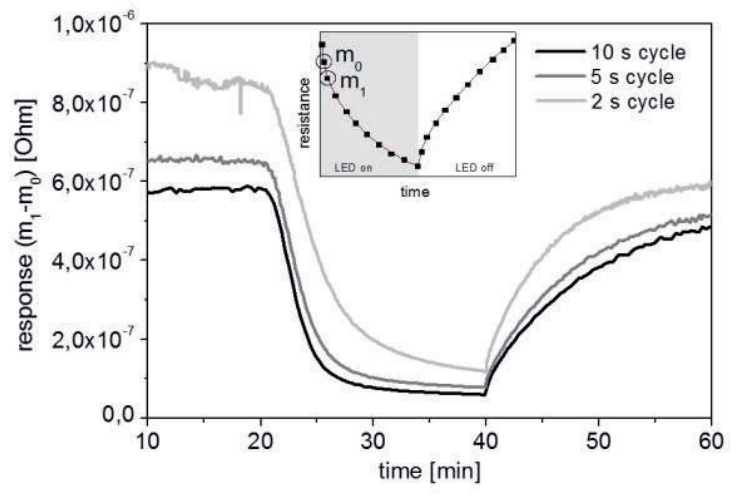

Fig. 3: Response vs. time of mesoporous $\ln _{2} \mathrm{O}_{3}$ particles under ozone exposure (500 ppb, $500 \mathrm{~mL} / \mathrm{min}$ flow rate, $20 \%$ relative humidity, $150{ }^{\circ} \mathrm{C}$ ) for different illumination times with blue light $(466 \mathrm{~nm})$. Inset: Measurement points for multi-signal evaluation.

The correlation between illumination time and sensor response can also be seen in the calculated $t_{90}$ reaction times of the sensor (see Tab. 1).

Tab. 1: Calculated t9o reaction times of the sensor for different illumination times.

\begin{tabular}{|c|c|}
\hline Illumination time [s] & t90, reaction $[\mathrm{min}]$ \\
\hline 2 & 10.3 \\
\hline 5 & 7.0 \\
\hline 10 & 6.3 \\
\hline
\end{tabular}

The illumination time of $10 \mathrm{~s}$ provides the best signal. This leads to the assumption that the time constant of the surface reaction of ozone molecules at the $\ln _{2} \mathrm{O}_{3}$ surface is in the same range.

In addition, the cycle shape is changing during gas exposure, which suggests that it contains additional information about the underlying mechanisms. Therefor further experiments will be carried out to study these mechanisms in more detail.

\section{Conclusion}

Mesoporous $\ln _{2} \mathrm{O}_{3}$ is a promising material for resistive gas sensing because it is highly sensitive to oxidizing gases like ozone even at low temperature. The kinetics of semiconducting gas sensors is typically slow without activation. Instead of thermal activation, optical activation of the $\ln _{2} \mathrm{O}_{3}$ particles can be used. Especially cyclic optical activation is a proper tool for improving the response and recovery times of the sensor.

In this work, we presented a systematic optimization process of the illumination time to study the influence of cyclic illumination and chemical surface reaction during ozone exposure on the nanoporous $\ln _{2} \mathrm{O}_{3}$ sensing layer. It was shown that the illumination time is correlated to the sensor response. The illumination time of $10 \mathrm{~s}$ provides the best signal which leads to the assumption that the time constant of the ozone reaction at the $\ln _{2} \mathrm{O}_{3}$ surface is in the same range.

All in all, the presented method is a proper tool to study surface reaction kinetics of ozone molecules at the $\ln _{2} \mathrm{O}_{3}$ surface. However, details about the underlying mechanisms are still under investigation.

\section{References}

[1] S. Bianchi, E. Comini, M. Ferroni, G. Faglia, A. Vomiero, G. Sberveglieri, Indium oxide quasimonodimensional low temperature gas sensor, Sensors and Actuators B: Chemical 118, 204207 (2006); doi: 10.1016/j.snb.2006.04.023

[2] E. Comini, G. Faglia, G. Sberveglieri, UV light activation of tin oxide thin films for $\mathrm{NO} 2$ sensing at low temperatures, Sensors and Actuators B: Chemical 78, 73-77 (2001); doi: 10.1016/S09254005(01)00796-1

[3] D. Klaus, D. Klawinski, S. Amrehn, M. Tiemann, T. Wagner, Light-activated resistive ozone sensing at room temperature utilizing nanoporous $\mathrm{In}_{2} \mathrm{O}_{3}$ particles: Influence of particle size, Sensors and Actuators B: Chemical 217, 181-185 (2015); doi: 10.1016/j.snb.2014.09.021

[4] T. Wagner, C.-D. Kohl, C. Malagù, N. Donato, M. Latino, G. Neri, M. Tiemann, UV light-enhanced $\mathrm{NO} 2$ sensing by mesoporous $\ln _{2} \mathrm{O}_{3}$ : Interpretation of results by a new sensing model, Sensors and Actuators B: Chemical 187, 488-494 (2013); doi: 10.1016/j.snb.2013.02.025

[5] T. Wagner, J. Hennemann, C.-D. Kohl, M. Tiemann, Photocatalytic ozone sensor based on mesoporous indium oxide: Influence of the relative humidity on the sensing, Thin Solid Films 520, 918-921 (2011); doi: 10.1016/j.tsf.2011.04.181

[6] D. Meixner, C. Kohl, T. Wagner, Photonic metal oxide gas sensors: low temperature ozone sensing by cyclic optical excitation of $\operatorname{In}_{2} \mathrm{O}_{3}$, Proceedings SENSOR 2015, AMA Conferences, 708 - 711, (2015); doi: 10.5162/sensor2015/E7.3

[7] A. Gramm, A. Schütze, High performance solvent vapor identification with a two sensor array using temperature cycling and pattern classification, Selected Papers from Eurosensors XVI, Sensors and Actuators B: Chemical 95 58-65 (2003); doi: 10.1016/S0925-4005(03)00404-0

[8] D. Klaus, S. Amrehn, M. Tiemann, T. Wagner, One-step synthesis of multi-modal pore systems in mesoporous $\ln _{2} \mathrm{O}_{3}$ : A detailed study, Microporous and Mesoporous Materials 188 133139 (2014); doi: 10.1016/j.micromeso.2014.01.00 